\title{
Développements des publications en psycho-oncologie en France
}

\author{
Developments concerning psycho-oncology publications in France
}

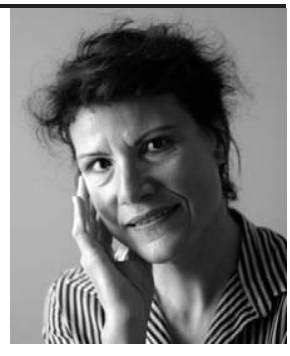

\author{
M.-F. Bacqué \\ (C) Springer-Verlag France 2010
}

Depuis 2002, la revue Psycho-Oncologie a considérablement évolué. L'idée a pris corps dans les suites dramatiques du 11 septembre 2001, alors que le congrès de la SFPO (Société française de psycho-oncologie) accueillait à Caen de nombreux collègues d'outre-Atlantique. La visite du mémorial dédié à la Seconde Guerre mondiale trouvait des échos indéniables chez les Américains et les Canadiens présents, à tel point que les visages marqués de ceux qui avaient vécu en direct l'attaque des tours avaient encore pâli en ressentant le parallèle entre la dramaturgie de l'avènement de la domination nazie et l'inquiétude engendrée par la démonstration des terroristes d'Al-Qaïda. Est-ce à la faveur d'une catastrophe envisagée que nos esprits ont franchi le pas de la création de Psycho-Oncologie ? L'idée était sans conteste en préparation ; cependant, un consensus s'est immédiatement dégagé et a été suivi d'une réalisation concrète dès le début de l'année 2002.

Des années sans aucun doute plus sereines allaient voir l'épanouissement de la revue. Les psychiatres et psychologues nord-américains ont été parrains et marraines de sa naissance. Aujourd'hui, ce n'est d'ailleurs pas sans humour qu'il est nécessaire de leur rappeler qu'ils n'ont pas été les premiers à écrire en psycho-oncologie... Si le terme de psycho-oncologue est effectivement anglo-saxon, il n'a pas été adopté complètement par les Français, tant s'en faut ! Nous avons bien compris qu'il s'agissait d'adjoindre une forme de spécialisation cancérologique à la discipline psychologique ; cependant, si la nature des différents cancers, si la singularité de leurs traitements, si la richesse et la particularité des représentations collectives et individuelles de ces maladies entraînent une connaissance particulière, la méthode clinique et son éthique, celles qui concourent à la rencontre avec une personne reste la même pour tous les patients.

M.-F. Bacqué $(\bowtie)$

Département de psychologie, université de Strasbourg,

12, rue Goethe, F-67000 Strasbourg, France

e-mail : mfbacque@club-internet.fr
Depuis 2002, nous avons affiné les diagnostics psychologiques et psychiatriques portés sur les patients atteints de cancer, dans le but de multiplier les approches de patients jadis oubliés, et surtout de trouver de meilleures indications thérapeutiques. Les nouveaux diagnostics portant sur les difficultés d'acceptation de la maladie, du traitement ou même de la guérison ont permis de relativiser la psychopathologie de patients qui présentaient des troubles de l'identité, des syndromes anxieux, dépressifs, parfois délirants en rapport avec leur cancer. Même si ces patients avaient une fragilité antérieure, l'irruption du cancer pouvait se traduire par un abandon dans la dépression, par un excès d'angoisse qui aboutissait à l'arrêt ou au contraire à un surtraitement, par un tel changement de leur image du corps tant la perte de repère amenait une perplexité incompatible avec le contact régulier avec l'équipe soignante.

La question diagnostique a donc été maintes fois posée au cours de numéros mémorables de la revue Psycho-Oncologie : les groupes (familles, malades, soignants), les différents types de cancer (cancers cérébraux, cancers génitaux, rectocoliques), les atteintes nouvelles (image du corps, troubles mentaux), les stades de la vie (adolescence, vieillissement), la politique de santé mais aussi l'injustice, l'inégalité, la précarité, les manifestations douloureuses, l'angoisse d'une grossesse débouchant sur le cancer, l'information, les représentations, la charge symbolique, la qualité de vie subjective... La prévention des cancers, mais aussi des abandons de traitement et des rechutes nous ont permis de tisser le schéma d'une meilleure prise en charge, et tout dernièrement de concevoir un numéro spécial sur les psychothérapies de patients atteints de cancer. L'accompagnement psychologique n'est plus ce suivi développé par des praticiens qui mettaient en place des entretiens spécialisés en fonction de leur rôle dans un service ou d'un transfert dû au hasard d'une visite. Les colonnes de Psycho-Oncologie ont été le lieu non seulement d'observations mais véritablement de l'élaboration d'une nouvelle façon de prendre en considération les patients atteints de cancer. 
La preuve en est aujourd'hui dans les colonnes de ce numéro électronique : les articles novateurs ont été si nombreux cette année que plutôt que de faire attendre nos auteurs jusqu'à l'année suivante, nous avons décidé de consacrer les articles qui ont été retenus par les experts à un supplément. Ce supplément électronique est en quelque sorte un test pour une parution plus fréquente de Psycho-Oncologie. Il est surtout le signe d'une créativité redoublée chez les auteurs qui, de plus en plus, mènent des recherches originales, apportent des observations précieuses, réfléchissent au système de santé et à l'évolution de notre société face au cancer.

Qu'ils soient remerciés pour leur participation à ce numéro exceptionnel qui, je le rappelle, présente les mêmes caractéristiques scientifiques que nos numéros habituels et les mêmes possibilités de figuration dans les bases de données internationales et d'accès en ligne. 